\title{
Hepatic Fibrosis: Novel Strategies in Detection and Therapy
}

\author{
Venkataramana Bhat, Mamatha Bhat*
}

\section{INTRODUCTION}

Hepatic cirrhosis is the final stage of progressive hepatic fibrosis, and is histologically characterized by collapse of hepatic lobules, formation of fibrous septae, and hepatocyte regeneration (1). Fibrosis as a scarring response to liver damage may be thought of as beneficial, since it contains the injurious process (2). Ultimately however, this progressive scarring can lead to impairment of liver function, development of hepatocellular carcinoma and portal hypertension with all its associated complications. Recently, there has been a growing understanding of the pathophysiology behind fibrosis, which has contributed to the development of agents that could potentially inhibit and even reverse the fibrotic process in the future. Using non-invasive means that are more precise, reproducible, and less fraught with complications would allow the clinician to monitor disease progression, clinical outcomes and response to antifibrotic treatment. In this review article, we will cover the various etiologies of hepatic fibrosis, the currently used diagnostic modalities, serum markers, and transient elastography as novel non-invasive diagnostic modalities, and potential agents that could be used in the future to halt or even reverse fibrosis.

\section{ETIOLOGIES OF HEPATIC FIBROSIS}

Hepatic cirrhosis can be the endpoint of various pathologic conditions; however, alcoholic liver disease and viral hepatitis $\mathrm{C}$ account for most cases of hepatic cirrhosis in Canada and the U.S. It is estimated that between 210,000 and 275,000 people are currently infected with the hepatitis $\mathrm{C}$ virus in Canada, with an additional 5000 people getting infected annually (3). Although there are no detailed epidemiological data

*To whom correspondence should be addressed:

Mamatha Bhat

2250 Guy Apt 908, Montreal, Quebec, Canada, H3H 2M3

Tel: 514-932-6926

Email: Mamatha.bhat@mail.mcgill.ca from Canada, it is estimated that 600,000 Canadians are chronically infected with hepatitis $\mathrm{B}$, the majority of whom are immigrants from endemic areas (4). Primary biliary cirrhosis $(\mathrm{PBC})$, primary sclerosing cholangitis (PSC), hemochromatosis, autoimmune hepatitis, nonalcoholic fatty liver disease (NAFLD), and Wilson's disease comprise the rest of the etiologies of hepatic cirrhosis.

\section{DIAGNOSIS OF HEPATIC FIBROSIS}

These conditions can be elucidated through specific biomarkers and antibody assays. Imaging techniques such as abdominal ultrasound and CT scanning are used to detect and follow structural changes in the liver parenchyma as it progresses from fibrosis to cirrhosis. Liver parameters such as size, appearance of its surface and margin, and the echogenicity of parenchymal texture can be estimated through abdominal ultrasound. However, compensated hepatic cirrhosis can be accurately diagnosed in 80 to $87 \%$ of patients $(5,6,7)$ with a specificity of $81.5 \%$ (7). This range of sensitivities is based on the following three studies: a prospective study by Gaiani et al. where 212 patients underwent liver biopsies as gold standard and concurrent ultrasonographic assessment (5), a study of 70 patients by Ferral et al.(6), and similar evaluation of 48 patients by Zheng et al (7). The presence of cirrhosis was inferred based on liver surface nodularity, relative enlargement of the caudate lobe as compared to the right lobe, splenomegaly and portal flow velocity. CT scan has been deemed to have a sensitivity of $84 \%$ and a specificity of approximately $100 \%$ for the detection of cirrhosis (8).

The gold standard to diagnose hepatic fibrosis and cirrhosis is liver biopsy. This is obtained via percutaneous, transjugular, radiographically-guided fine-needle or laparoscopic route, depending upon the clinical setting. The sensitivity of the blind percutaneous liver biopsy when compared to the ultimate gold standard, which is a sizeable biopsy 
obtained laparoscopically, is $82 \%$ (9). Histopathological examination enables the clinician to grade the severity of necroinflammation and stage the extent of fibrosis. The Metavir scoring system attributes a score to the stages of fibrosis on a $0-4$ scale as follows (10): $\mathrm{F} 0=$ no fibrosis; F1 = portal fibrosis without septa; F2 = portal fibrosis and few septae; F3 = numerous septae without cirrhosis; F4 = cirrhosis.

Liver biopsy is an invasive and costly procedure, and samples only a small portion of the liver. Thus, it cannot afford a global assessment of hepatic fibrosis, and is subject to sampling variation and inter- and intraobserver error. In addition, liver biopsy is associated with a significant morbidity of $3 \%$ and a mortality rate of $0.03 \%$ (11). Potential complications include local hematoma, infection, and pain related to the procedure.

\section{SERUM MARKERS}

Serum markers to monitor the evolution of liver disease are appealing, because they are non-invasive, and repeated testing at regular intervals is more feasible due to lower cost (12). Panels of blood markers have been weighed against the gold standard of liver biopsy, mostly in patients with chronic hepatitis $\mathrm{C}$ or cirrhosis due to viral hepatitis C. A meta-analysis of these studies revealed that serum markers can rule in or rule out fibrosis in approximately $35 \%$ of patients (13). However, when looking at patients individually, these markers could not reliably differentiate between the various stages of fibrosis. A more recent study by Sebastiani et al. incorporated three panels of serum markers to devise an algorithmic approach that improved diagnostic accuracy (14). The three panels evaluated were the APRI (aspartate transaminase to platelet ratio index), the Forns' index (platelets, gammaglutamyltranspeptidase, cholesterol) and the Fibrotest (GGT, haptoglobin, bilirubin, apolipoprotein A, alpha2-macroglobulin). An algorithm consisting of the APRI followed by the Fibrotest boosted the diagnostic accuracy of fibrosis to above $90 \%$. This group estimated that use of this algorithm could obviate the need for up to $50 \%$ of liver biopsies. However, the individual stages of fibrosis are not distinguishable using this algorithm. Serum markers are just beginning to be used in clinical practice in order to follow patients with hepatitis of viral etiology, as these are not proven in other types of liver disease. The limitation of these serum markers is the possibility of false positives when there is highly active hepatic inflammation.

\section{FIBROSCAN}

Fibroscan is an innovative approach to staging hepatic fibrosis based on elastography, which provides rapid measurement of mean hepatic tissue stiffness (15). A probe is employed to transmit a vibration of low frequency and amplitude into the liver. This vibration wave triggers an elastic shear wave, whose velocity through the liver is directly proportional to tissue stiffness measured in kilopascals (kPa). The Fibroscan technique can measure liver stiffness of a volume that is 100 times greater in size than a standard liver biopsy, thus giving a better overall picture of fibrosis in hepatic parenchyma. Validation studies have reported excellent intra- and inter-operator correlation, and have shown the various degrees of liver stiffness to reliably correlate with the stages of fibrosis $(15,16)$. Sensitivity of the Fibroscan technique ranged from 79 to $95 \%$, and specificity from 78 to $95 \%$, compared to the gold standard of liver biopsy. The limitations of this technique are attenuation of elastic waves in fluid or adipose tissue, which would impair assessment of fibrosis in patients with ascites or morbid obesity. The problem of obesity is especially rampant in North American society, with $30 \%$ of the population being overweight or obese.

Use of Fibroscan in conjunction with serum markers of fibrosis (Fibrotest) further enhances accuracy in the staging of fibrosis as reported by Castera et al (17). In this study, there was concordance in staging between Fibroscan and Fibrotest in $70-80 \%$ of patients. Combined use of the Fibroscan and Fibrotest assay in these instances resulted in concordance with liver biopsy as follows: $84 \%$ for significant fibrosis $(\mathrm{F} \geq 2)$, $95 \%$ for severe fibrosis $(\mathrm{F} \geq 3)$, and $94 \%$ for cirrhosis ( $\mathrm{F}$ $=4)$. Fibroscan is, however, an expensive instrument with an approximate cost of US\$80 000. It is currently in the process of being approved for use in Canada.

\section{EMERGING THERAPIES AGAINST HEPATIC FIBROSIS}

Patients with active ongoing hepatic inflammation are often not seen until fibrosis has occurred, as they are asymptomatic most of the time. However, if tests were performed to catch these patients early on the basis of increased liver enzymes, there is the potential to curtail or even reverse hepatic fibrosis. Some agents that stem the development of hepatic fibrosis are already in use. Agents that can reverse fibrosis, however, are only at the investigational stage. Targetting various aspects of fibrotic process would likely have a tremendous impact on the morbidity and mortality in these patients.

The removal of injurious stimuli is a strategy already in use, in the form of antiviral therapy for hepatitis, copper chelation for Wilson's disease, phlebotomy for hemochromatosis, and discontinuation of hepatotoxic medication (18).

Corticosteroids have been used successfully to suppress hepatic inflammation in autoimmune and 
alcoholic hepatitis (19). Ursodeoxycholic acid has been proven to increase survival in $\mathrm{PBC}$ patients by binding bile acids, and thus also decreasing hepatic inflammation (20). Neutralizing inflammatory cytokines with specific receptor antagonists (TNFalpha, IL-1 receptor antagonists) and prostaglandin E have been tested in murine models, but not yet in humans (21). Whether colchicine, an anti-mitotic agent, has any antifibrotic activity is still controversial, due to conflicting study results $(22,23)$.

Another attractive target in curtailing hepatic fibrosis is the downregulation of hepatic stellate cell activation. Interferon gamma is already used in combination with ribavirin for therapy of hepatitis $\mathrm{C}$ infection. It is postulated that the antifibrotic effects of the interferons may be partially related to downregulation of stellate cell activation. This mechanism could explain the improvement in fibrosis described in patients with viral hepatitis $\mathrm{C}$ who do not have a virologic response to interferon alpha (24). Trials of antioxidants (nacetylcysteine, alpha-tocopherol) are currently underway in humans. Angiotensin II receptors are upregulated in stellate cell activation, thus angiotensin converting enzyme inhibitors and angiotensin receptor blockers have demonstrated antifibrotic activity in vitro and in animals. This has yet to be replicated in humans (25). Hepatic growth factor antagonist and collagen synthesis inhibitors (TGF-B antagonist) have shown promise in animal trials as well (26).

Promoting matrix degradation through matrix metalloproteinases is an antifibrotic strategy shown to be beneficial in a murine model (27). Specific apoptosis of hepatic stellate cells is another interesting theoretical idea, but has not yet been investigated (28).

\section{CONCLUSION}

The future of hepatic fibrosis detection and treatment appears bright with novel markers and investigational agents on the horizon. Optimizing the accuracy of noninvasive testing via serum assays and elastography is essential to allowing wider implementation of these tests in clinical practice. When optimized, these tests will be able to provide an 'integrated' readout of liver activity, rather than the limited sampling of a conventional liver biopsy. Early detection of fibrosis, and regular monitoring of fibrosis, would allow for initiation of anti-fibrotic therapies capable of halting and even reversing this process. This would in turn prevent progression to hepatic cirrhosis, and the morbidity and mortality this condition entails. The development of these various early fibrosis detection techniques bodes well for the future care of patients with liver disease.

\section{REFERENCES}

1. Gressner, AM. The cell biology of liver fibrogenesis - an imbalance of proliferation, grow th arrest and apoptosis of myofibroblasts. Cell Tissue Res 1998; 292:447.

2. Poynard T, Mathurin, P, Lai, CL, et al. A comparison of fibrosis progression in chronic liver diseases. J Hepatol 2003; 38:257.

3. Zou, S., Tepper, M. \& Giulivi, A. (2000). Current status of hepatitis C in Canada. Canadian Journal of Public Health, 91, 1, S10-S15.

4. Bloodborne Pathogens Section, Blood Safety Surveillance and Health Care Acquired Infections Division, Health Canada, 2003

5. Gaiani S, Gramantieri L, Venturoli N et al. What is the criterion for differentiating chronic hepatitis from compensated cirrhosis? A prospective study comparing ultrasonography and percutaneous liver biopsy. J Hepatol 1997; 27:979-985.

6. Ferral H, Male R, Cardiel R, et al. Cirrhosis: Diagnosis by liver surface analysis with high-frequency ultrasound. Abdominal Imaging 1992;17: 1432-0509

7. Zheng R, Wang Q, Lu M, et al. Liver fibrosis in chronic viral hepatitis: an ultrasonographic study. World J Gastroenterol. 2003; 9:2484-2489.

8. Fidler, J.L. \& Stark, D.D. (1999). Magnetic resonance imaging of the upper abdomen. In J. Traveras \& J. Ferrucci (Eds.), Radiology: diagnosis - imaging - intervention, vol. 4 (pp. 1-17). Pennsylvania: J.B. Lippincott Company.

9. Pagliaro L, Rinaldi F, Craxi A, et al. Percutaneous blind biopsy versus laparoscopy with guided biopsy in diagnosis of cirrhosis. Dig Dis Sci. 1983; 28:39-43.

10. P. Bedossa, T. Poynard and The METAVIR cooperative study group. An algorithm for the grading of activity in chronic hepatitis C, Hepatology 1996; 24:289-293

11. Garcia-Tsao G. Boyer JL. Outpatient liver biopsy: how safe is it? Ann Intern Med 1993; 118: 150-3.

12. de Franchis R, Dell'Era A. Non-invasive diagnosis of cirrhosis and the natural history of its complications. Best Practice \& Research Clinical Gastroenterology 2007; 21:3-18.

13. Sebastiani G, Vario A, Guido $M$ et al. Stepwise combination algorithms of non-invasive markers to diagnose significant fibrosis in chronic hepatitis C. J Hepatol 2006; 44: 686-693.

14. Parkes J, Guha IN, Roderick P et al. Performance of serum marker panels for liver fibrosis in hepatitis C. J Hepatol 2006; 44: 462-474.

15. Ziol $\mathrm{M}$, et al. Noninvasive assessment of liver fibrosis by measurement of stiffness in patients with chronic hepatitis $\mathrm{C}$. Hepatology 2005; 41:48-54.

16. Ganne-Carrie N, Ziol M, de Ledinghen V, et al. Accuracy of liver stiffness measurement for the diagnosis of cirrhosis in patients with chronic liver diseases. Hepatology 2006; 44:1511-1517.

17. Castera L, Vergniol J. Prospective comparison of transient elastography, Fibrotest, APRI, and liver biopsy for the assessment of fibrosis in chronic hepatitis C. Gastroenterology 2005; 128:343-350.

18. Bonis PA, Friedman SL, Kaplan MM. Is liver fibrosis reversible? N Engl J Med 2001; 344:452.

19. Czaja A, Freese DK. Diagnosis and Treatment of Autoimmune hepatitis. Hepatology 2003; 473-496

20. Poupon RE, Lindor KD, Cauch-Dudek K, et al. Combined analysis of randomized controlled trials of ursodeoxycholic acid in primary biliary cirrhosis. Gastroenterology 1997; 113:884.

21. Bruck R, Shirin H, Hershkoviz R, et al. Analysis of Arg-GlyAsp mimetics and soluble receptor of tumour necrosis factor as therapeutic modalities for concanavalin A induced hepatitis in 
mice. Gut 1997; 40:133.

22. Kershenobich D, Vargas F, Garcia-Tsao G, et al. Colchicine in the treatment of cirrhosis of the liver. N Engl J Med 1988; 318:1709.

23. Rodriguez L, Cerbon-Ambriz J, Munoz ML. Effects of colchicine and colchiceine in a biochemical model of liver injury and fibrosis. Arch Med Res 1998; 29:109.

24. Poynard T, Marcellin P, Lee SS, et al. Randomised trial of interferon alpha $2 \mathrm{~b}$ plus ribavirin for 48 weeks or for 24 weeks versus interferon alpha $2 \mathrm{~b}$ plus placebo for 48 weeks for treatment of chronic infection with hepatitis $\mathrm{C}$ virus. International Hepatitis Interventional Therapy Group (IHIT).
Lancet 1998; 352:1426

25. Jonsson JR, Clouston AD, Ando Y, et al. Angiotensin-converting enzyme inhibition attenuates the progression of rat hepatic fibrosis. Gastroenterology 2001; 121:148.

26. Ueki T, Kaneda Y, Tsutsui H, et al. Hepatocyte growth factor gene therapy of liver cirrhosis in rats. Nat Med 1999; 5:226.

27. Iimuro $\mathrm{Y}$, Nishio $\mathrm{T}$, Morimoto $\mathrm{T}$, et al. Delivery of matrix metalloproteinase-1 attenuates established liver fibrosis in the rat. Gastroenterology 2003; 124:445.

28. Gressner AM. The cell biology of liver fibrogenesis - an imbalance of proliferation, growth arrest and apoptosis of myofibroblasts. Cell Tissue Res 1998; 292:447.

Venkataramana Bhat (MBBS) is a Research fellow at the Douglas Hospital Research center (McGill University, Montreal, Canada).

Mamatha Bhat (BSc, MDCM 2005) is a third-year Internal Medicine resident at the Royal Victoria Hospital (McGill University, Montreal, Canada). She will be starting her fellowship in Gastroenterology at McGill this year, and has a special interest in Hepatology. 